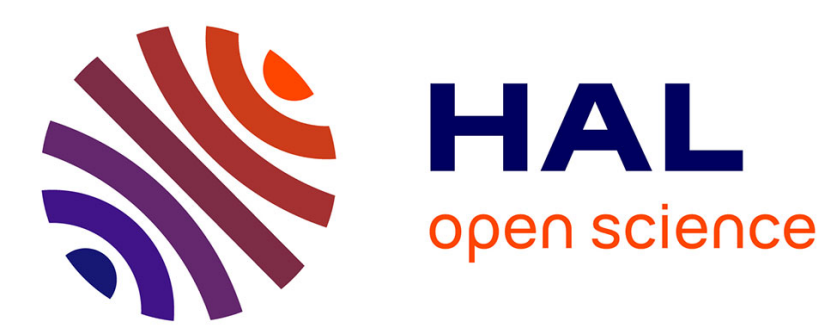

\title{
Ultrafast electron dynamics and orbital-dependent thermalization in photoexcited metals
}

E. Silaeva, Emile Bévillon, R. Stoian, Jean-Philippe Colombier

\section{To cite this version:}

E. Silaeva, Emile Bévillon, R. Stoian, Jean-Philippe Colombier. Ultrafast electron dynamics and orbital-dependent thermalization in photoexcited metals. Physical Review B: Condensed Matter and Materials Physics (1998-2015), 2018, 98 (9), pp.094306. 10.1103/physrevb.98.094306 . ujm-02003534

\section{HAL Id: ujm-02003534}

\section{https://hal-ujm.archives-ouvertes.fr/ujm-02003534}

Submitted on 1 Feb 2019

HAL is a multi-disciplinary open access archive for the deposit and dissemination of scientific research documents, whether they are published or not. The documents may come from teaching and research institutions in France or abroad, or from public or private research centers.
L'archive ouverte pluridisciplinaire HAL, est destinée au dépôt et à la diffusion de documents scientifiques de niveau recherche, publiés ou non, émanant des établissements d'enseignement et de recherche français ou étrangers, des laboratoires publics ou privés. 


\title{
Ultrafast electron dynamics and orbital-dependent thermalization in photoexcited metals
}

\author{
E. P. Silaeva, E. Bevillon, R. Stoian, and J. P. Colombier* \\ Univ Lyon, UJM-St-Etienne, CNRS, Institute of Optics Graduate School, Laboratoire Hubert Curien UMR 5516, \\ F-42023 Saint-Etienne, France
}

(Received 5 June 2018; published 24 September 2018)

\begin{abstract}
We use a time-dependent density functional theory (TDDFT) to analyze nonequilibrium dynamics of laserexcited electrons in transition metals $(\mathrm{Ni}, \mathrm{Cr}, \mathrm{Cu})$ and shed light on the ultrafast thermalization process from a microscopical point of view. As a first result, after instant increase of the electron temperature up to $50000 \mathrm{~K}$, we observe that the dynamics of electron density of states is faster than a laser subcycle, on the attosecond timescale. This is related to an ultrafast rearrangement of excited electrons in space to accommodate strong changes in ion screening. Secondly, we show that the electron thermalization dynamics strongly depends on the electronic structure of a given metal. Excited by a 7-fs laser pulse, $d$-block transition metals exhibit two subsystems of electrons, each one achieving its own temperature. Due to a higher localization, electrons from $d$ block stay cold, while excited delocalized $s p$ electrons rapidly reach a high temperature. Electrons of each band of energy are mutually thermalized within the time of the laser pulse. Much more time is however needed to reach equilibrium of the whole electronic system. These results redraw the validity limits of current two-temperature models during the laser irradiation time, potentially impacting further material reaction.
\end{abstract}

DOI: 10.1103/PhysRevB.98.094306

\section{INTRODUCTION}

Ultrafast laser pulses are capable of precisely depositing and confining light energy during interaction with materials on a timescale much faster than thermal energy exchange between photoexcited electrons and lattice ions. This has a great potential for highly precise material micro- and nanoprocessing for a wide range of applications [1-3]. The characteristics and quality of the laser-induced micro- and nanostructures depend strongly on the electron dynamics and its influence on the transient properties of the materials during the first femtoseconds of the interaction with the laser pulse [4]. Control of photoexcited electron phenomena in solids on attosecond time scale also opens the door to petaherz optoelectronics [5] and attosecond metrology of solids [6].

When an ultrashort laser pulse irradiates a metal, light energy is absorbed by the electronic system which is driven out of thermal equilibrium. Due to electron-electron scattering nonthermal electrons relax to a new thermodynamic equilibrium characterized by a Fermi-Dirac energy distribution with an increased effective temperature. This process is called electron thermalization and its characteristic time can vary significantly between several femtoseconds up to several picoseconds depending on pulse duration, intensity, and material [7-9]. The laser-excited electrons interact with phonons and the laser energy is transferred from electrons to the lattice causing lattice heating and crystal structure change. The process of electron-phonon coupling and subsequent lattice modification depend strongly on the transient state of the electron system.

\footnotetext{
*jean.philippe.colombier@univ-st-etienne.fr
}

At relatively long and intense laser pulses, the thermalization of nonequilibrium electrons is expected to happen immediately compared to the characteristic time of electronphonon energy exchange. As a result, the electron temperature stays above the lattice temperature during several picoseconds and the framework of a two-temperature model (TTM) can be used to describe laser-induced processes in metals $[10,11]$. Beyond a standard TTM, a fast electron thermalization is also assumed to describe the effect of high electron temperature on electronic, optical and thermal properties of metals [12-16]. The modification of electronic structure due to increase of the effective electron temperature can change the metal melting temperature $[17,18]$, induce solid-solid phase transformation [19-22], and affect electron-phonon coupling dynamics $[23,24]$. It is not clear however if the modification of electronic structure is as fast as the electron thermalization. Additionally, the electron thermal state defined by a single temperature is usually considered to be non-orbital-selective but transient subband effects are likely to occur on short timescales.

A rather long electron thermalization time can be observed at lower laser irradiation intensities [25]. In this case, the nonequilibrium electron dynamics becomes important and limits the applicability of TTM. Additionally, the pumpprobe ellipsometry measurements report the change of the absorption and refractive index of a photoexcited metal that is usually associated with the presence of thermalized electron subsystem at high temperature during the laser pulse [26,27]. The assumption that electrons thermalize and affect the material optical properties and laser-matter interaction during a short laser pulse of less than 100-fs duration is however questionable. It seems more likely that the observed changes of optical properties are related to nonequilibrium electron dynamics rather than to the established effective temperature 
of electrons $[15,28]$. The nonequilibrium electron dynamics also significantly affects the laser-induced phase transitions. It was shown for example that highly excited nonequilibrium electrons can lead to ultrafast nonthermal melting of materials indicating that atomic bonds are softened [29]. To correctly define the limits of validity of TTM and explore the role of nonequilibrium electrons in laser-induced material modification, the electron thermalization process has to be elucidated.

The time scales of electron thermalization in metals as a function of laser fluence was extensively studied using Boltzmann transport equations [30]. This pertains to mostly low fluences and thus low electronic excitation levels. At higher excitation levels, typical for laser processing of metals, the electronic structure (i.e., density of states, chemical potential, etc.) is modified during the thermalization process and standard Boltzmann approach assuming constant density of states is not suitable anymore. Recently, the Boltzmann approach was combined with first-principles calculations to take into account these modifications of electronic structure and its importance in the interaction of nonequilibrium electrons with lattice was revealed [31]. First-principles calculations were done using density functional theory (DFT) that assumes adiabatic change of electronic distribution and thus limited to a rather slow electron thermalization process. Many-body perturbation theory $(\mathrm{GW}+\mathrm{BSE})$ is efficient when photoexcited steady states have to be self-consistently determined but it does not explicitly describe the transient evolution. A timedependent first-principles approach is needed to overcome these limitations and properly capture photoexcitation and ultrafast nonequilibrium dynamics of laser-excited electrons.

Time-dependent density functional theory (TDDFT) is widely used to simulate ultrafast laser-induced electron dynamics of isolated molecules and atomic clusters. There also exists a limited number of works related to TDDFT of laserexcited solids $[5,32,33]$. The TDDFT is the only computationally feasible ab initio method to treat the effects of strong transient fields on systems containing many electrons. TDDFT describes an excited quantum system as a time-dependent noninteracting Kohn-Sham state. Thus, it is limited in representing a path to thermalization because a thermal state is a mixed state. That is why TDDFT is usually applied to describe phenomena much faster than electron thermalization time $[34,35]$. Recently it was shown however that TDDFT could indirectly provide a mechanism for the electrons to thermally equilibrate with each other [36]. Moreover the motion of the atoms produce a large number of small electronic transitions affecting the evolution of the electron energy distribution function similar to a thermalization process [34,37-40]. For the same reason the external electric potential of a laser pulse contributes to the thermalization of electrons through the photon-electron-ion collisional process and thus TDDFT can reasonably capture the thermalization process during the laser pulse.

In the present work, we apply TDDFT to analyze electron thermalization dynamics in different metals excited by a femtosecond laser pulse. First, we explore an evolution of electronic density of states (DOS) of nickel (Ni) after its electron subsystem is instantly heated to $T_{e}=50000 \mathrm{~K}$. This temperature corresponds to typical ablation regimes. The change of the DOS as a result of "hot"-electron density spatial redistribution happens on a subfemtosecond time scale. Then, we analyze the behavior of electron occupation number in laser-excited transition metals $[\mathrm{Ni}$, chromium $(\mathrm{Cr})$, and copper $(\mathrm{Cu})]$ as a function of laser intensity. After a 7-fs laser pulse two subsystems of electrons thermalized at two different temperatures can be observed in $\mathrm{Ni}$ and $\mathrm{Cu}$. Localized electrons in $d$ block of these metals stay rather cold while the temperature of free $s p$ electrons significantly increases due to laser excitation. Both temperatures increase with laser intensity. Additionally, at higher laser intensity the fraction of the hot electrons is higher. This difference in temperature is much less pronounced in $\mathrm{Cr}$ as $d$ band is only partially filled and nearly fully overlaps $s p$ band around the Fermi level. The relevance of TDDFT approach in given calculations is also discussed.

The paper is organized as follows. In Sec. II we describe the calculation methods of TDDFT in real-time domain and models of metals illuminated by a laser pulse. In Sec. III the calculation results of electron thermalization dynamics in laser-excited metals are presented. Conclusions are drawn in Sec. IV.

\section{FORMALISM}

The evolution of laser-excited electron distribution in metals is calculated using time-dependent density functional theory (TDDFT) [41,42] as implemented in Octopus code [43]. In this real-time TDDFT the laser-induced electron-excitation process is described by time-dependent Kohn-Sham equations:

$$
i \hbar \frac{\partial}{\partial t} \psi_{i}(\mathbf{r}, t)=H_{\mathrm{KS}}(\mathbf{r}, t) \psi_{i}(\mathbf{r}, t)
$$

where $\psi_{i}(\mathbf{r}, t)$ is an orbital wave function of a single-electron state, and $i$ denotes the state index and runs over all states. The Kohn-Sham Hamiltonian $H_{\mathrm{KS}}(\mathbf{r}, t)$ is given by

$$
\begin{aligned}
H_{\mathrm{KS}}(\mathbf{r}, t)= & \frac{1}{2 m}\left(-i \nabla+\frac{e}{c} \mathbf{A}(t)\right)^{2}+v_{\text {ion }}(\mathbf{r}) \\
& +e^{2} \int d \mathbf{r}^{\prime} \frac{\rho(\mathbf{r}, t)}{\left|\mathbf{r}-\mathbf{r}^{\prime}\right|}+v_{\mathrm{xc}}\left(\mathbf{r}^{\prime}, t\right),
\end{aligned}
$$

where $e$ is an electron charge, $m$ is its mass, $v_{\text {ion }}(\mathbf{r})$ is the electron-ion potential, and $v_{\mathrm{xc}}(\mathbf{r}, t)$ is the exchangecorrelation potential. The Troullier-Martins pseudopotential is used for electron-ion interaction [44] and the PerdewBurke-Ernzerhof (PBE) functional is employed for exchangecorrelation potential [45]. The third term on the right side is the so-called Hartree energy $\left(E_{H}\right)$ describing electronelectron interaction where electron density $\rho(\mathbf{r}, t)$ is given by

$$
\rho(\mathbf{r}, t)=\sum_{i} f_{i}^{0}\left|\psi_{i}(\mathbf{r}, t)\right|^{2},
$$

where $f_{i}^{0}$ is the initial occupation number of a state $i$. The spatially-uniform electric field of the laser pulse is introduced through vector potential $\mathbf{A}(t)$ :

$$
\mathbf{A}(t)= \begin{cases}-c \frac{E_{0}}{\omega} \cos (\omega t) \sin ^{2}\left(\pi t / \tau_{\mathrm{L}}\right) & \text { if } 0<t<\tau_{L} \\ 0 & \text { otherwise, }\end{cases}
$$


where $\omega$ is the laser frequency corresponding to a wavelength of $\lambda=800 \mathrm{~nm}$ and time parameter $\tau_{\mathrm{L}}=18 \mathrm{fs}$ corresponds to the pulse full width at half maximum of $7 \mathrm{fs}$. The peak electric field is defined by $E_{0}=\sqrt{2 I_{\text {las }} /\left(c \varepsilon_{0}\right)}$ where $I_{\text {las }}$ is the peak intensity of the laser pulse in vacuum. It is, thus, assumed that the model describes the laser-matter interaction inside a skin layer of the metal where electric field amplitude is comparable to the one in vacuum. The vector potential $\mathbf{A}$ is parallel to the $x$ axis with $y$ and $z$ components being zero.

In Octopus code, Eqs. (1)-(3) are solved in real space with an enforced time-reversal symmetry method for time propagation. The orbital wave functions are represented on a three-dimensional spatial grid and calculated in a crystal unit cell. The reciprocal $k$-space cell is also discretized. The parameters of the numerical scheme were optimized to save the computational resources without losing the convergence of the results. The number of symmetry-reduced $k$ points is 165. The grid spacing of the spatial grid is taken to be $0.16 \AA$, considering the lattice constants of $3.5 \AA$ for $\mathrm{Ni}, 3.6 \AA$ for $\mathrm{Cu}$, and $2.9 \AA$ for $\mathrm{Cr}$. There are four atoms in the unit cells of face-centered cubic $\mathrm{Ni}$ and $\mathrm{Cu}$, and two atoms in the unit cell of body-centered cubic Cr. Only valence electrons in the spin unpolarized state are considered in the calculations to reduce the computational cost. Spin polarization has a negligible effect on the results presented in the paper according to our tests. A time step of 1 is used. Atoms are fixed in their equilibrium positions, since for considered time delays ( $10 \mathrm{fs}$ ) the slow atomic movements are not expected to affect the electron dynamics.

We consider the evolution of the electron occupation number and DOS that have crucial impact on metal heat capacity, electric and thermal conductivity, electron-phonon coupling, and other properties sensible to the changes of electronic structure [12]. The fractional electron occupation number of an energy level $i$ is calculated as follows

$$
f_{i}(t)=\sum_{j} f_{j}^{0}\left|\left\langle\phi_{i} \mid \psi_{j}(t)\right\rangle\right|^{2},
$$

where $\psi_{j}$ are the solutions of Eq. (1). $\phi_{i}$ are the ground-state wave functions satisfying the eigenvalue equations

$$
H_{\mathrm{KS}}^{t=0} \phi_{i}=\epsilon_{i}^{t=0} \phi_{i},
$$

that are solved using self-consistent-field (SCF) method. The time-dependent DOS can be obtained from the ensemble of $\epsilon_{i}(t)$ by solving eigenvalue equations at each moment of time for the time-dependent wave functions $\psi_{i}(t)$ :

$$
H_{\mathrm{KS}}(t) \psi_{i}(t)=\epsilon_{i}(t) \psi_{i}(t)
$$

\section{RESULTS AND DISCUSSIONS}

Most of the valence electrons of the considered transition metals are strongly localized in a $d$ block. In most cases, these are accessible to excitation at the current laser wavelengths. During the laser excitation the electrons transit from these $d$ orbitals to $s p$ orbitals where they become nearly free. It was previously reported that high electron excitation levels and depopulation of the $d$ block lead to the change of the electron-orbital eigenvalues and thus of the DOS [12]. The electron excitation corresponds to the change of the electron occupation numbers. The dependence of an eigenvalue of an orbital on its occupation is related to the energy exchange between interacting electrons. When an electron is removed from an orbital $i$, i.e., when $f_{i}$ becomes 0 , the remaining orbitals are drawn closer to the nucleus, which becomes more effectively screened [46].

Upon ultrafast laser excitation, the dynamics of the electronic structure can occur through two relaxation channels, the density of states reorganization and the redistribution of the electron number. To unravel the two effects, we discuss first the case of a thermal excitation assuming an instantaneously thermalized electron population with no laser field perturbation but a fixed occupation number representative of $T_{e}$. In a second time the time-dependent electron distribution is investigated with a vector potential corresponding to a laser pulse at different intensity values.

\section{A. Ultrafast dynamics of the DOS response}

To analyze the dynamics of the electron energy equilibration, we instantly change the electron occupation number of the Ni ground state and then follow the evolution of its density of states. First, we calculate the ground state of $\mathrm{Ni}$ at $0 \mathrm{~K}$, using SCF method in Eq. (6), where electron occupation number as a function of energy follows Fermi-Dirac distribution:

$$
f_{i}^{0}=\frac{1}{1+\exp \left(\frac{\epsilon_{i}-\mu\left(T_{e}\right)}{k_{b} T_{e}}\right)},
$$

where $\mu$ is the chemical potential of a metal that is equal to the Fermi energy at zero electron temperature $T_{e}=0 \mathrm{~K}$. Then, the obtained ground-state wave functions are used as an initial condition for a TDDFT calculation of Eqs. (1)-(3) without external vector potential $\mathbf{A}=0$ but with modified occupation number $f_{i}^{0}$. At $t=0, f_{i}^{0}$ follows Eq. (8) at high electron temperature $T_{e}=50000 \mathrm{~K}$. This initial condition corresponds to a Dirac excitation of electrons through strong heating. The temporal evolution of the DOS adapting to such changes in electron occupation number is shown in Fig. 1(a).

At zero temperature, the DOS of $\mathrm{Ni}$ has a well defined peak corresponding to the $d$-block states. When at $t=0$ the occupation number is changed to follow the Fermi-Dirac distribution at $50000 \mathrm{~K}$, the $d$ block becomes strongly depopulated and moves closer to the ion due to a modified ion screening. This also leads to the strong change of electronelectron interaction resulting in a large drop of Hartree energy [see inset of Fig. 1(b)]. Instantaneously, the electron system is pushed out of equilibrium by the DOS reorganization as can be seen from the occupation number at $t=0$ shown in Fig. 1(b). In the calculation, a time-dependent set of wave functions $\left|\psi_{i}(t)\right\rangle$ evolve from the given initial ones, modifying both $f_{i}(t)$ and the time-evolving density in the Hartree energy term as indicated by Eqs. (2) and (3). Actually, the FermiDirac occupation numbers, calculated taking into account the ground-state eigenvalues, as a function of new instantly changed eigenvalues, deviate from an equilibrium FermiDirac distribution. It takes 50 as more for the electrons to reorganize and reach an equilibrium state at $50000 \mathrm{~K}$. At equilibrium, the result of TDDFT calculation coincides with the result of SCF calculation that can be obtained using Eqs. (6), (3), and (8) at $50000 \mathrm{~K}$. The change in Hartree energy 


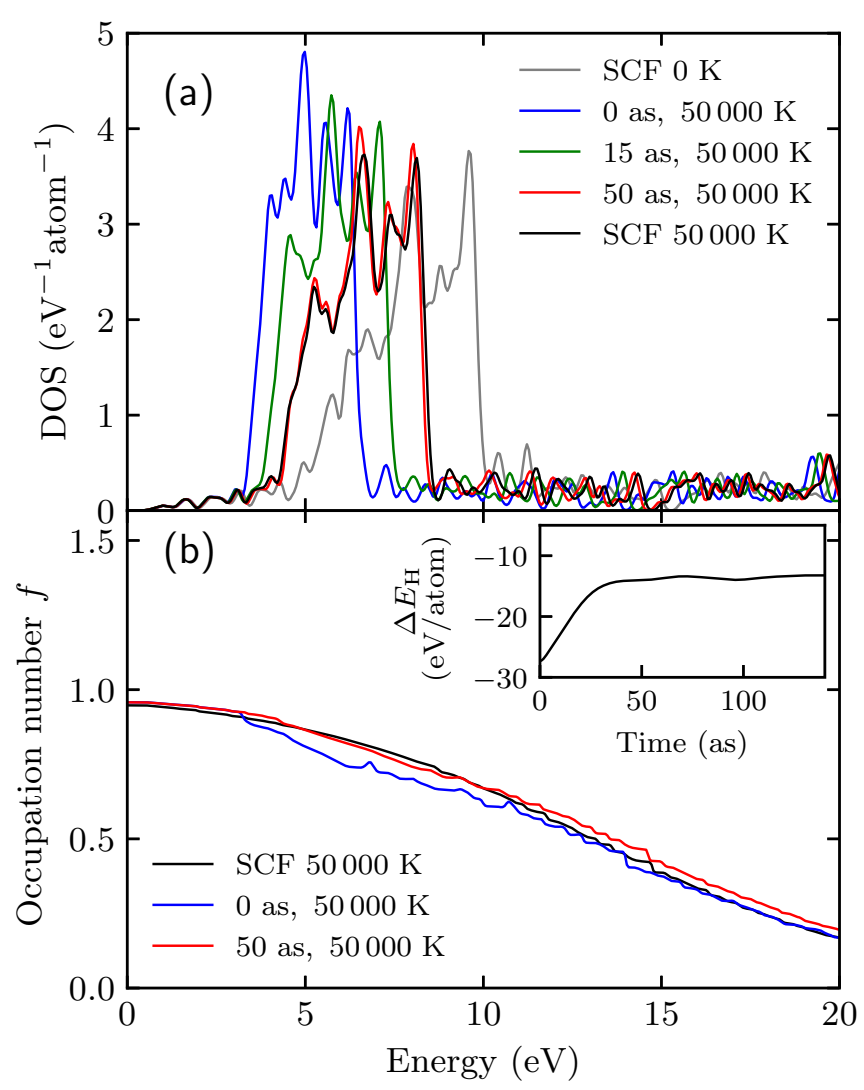

FIG. 1. Evolution of the density of states (a) and electron occupation number (b) as a result of instant heating of an electron subsystem in Ni up to $50000 \mathrm{~K}$. (a) After heating (blue curve) the DOS peak instantly shifts to the lower energies compared to its initial position at $0 \mathrm{~K}$ (gray curve) and then gradually moves back to the higher energies (green curve) until it converges (red curve) with the equilibrium DOS at $50000 \mathrm{~K}$ obtained through SCF calculation (black curve). (b) Electron occupation number $f$ transiently deviates from equilibrium. The inset in (b) shows the corresponding evolution of Hartree energy change $\Delta E_{H}$ compared to its value at $0 \mathrm{~K} . \Delta E_{H}$ is initially very high and then decreases and saturates at the same value that can be obtained from SCF calculation at $50000 \mathrm{~K}$.

describing electron-electron interaction between the ground and excited state is first rather high and then it decreases also reaching the same value as obtained in SCF calculation at $50000 \mathrm{~K}$. The convergence of the nonequilibrium electron dynamics described by TDDFT towards an equilibrium state obtained using SCF approach is a result of electron-electron relaxation processes implicitly included in TDDFT.

The observed changes in DOS, occupation number, and Hartree energy are related to the electron spatial redistribution. The change in electron density compared to the ground state at $0 \mathrm{~K}$ around $\mathrm{Ni}$ atoms are shown in Fig. 2. At $t=0$, the electron density of $d$ orbitals located near the nuclei decreases and the density of free $s p$ electrons located between the atoms increases. A redistribution of electrons inside the $d$ orbitals also takes place as the $d$ block is not fully filled (configuration of valence electrons in $\mathrm{Ni}$ atoms is $3 d^{8} 4 s^{2}$ ). The electron density of $d_{z^{2}}$ orbital increases with a twofold symmetry of the electron density change near the nuclei. Later on the $s p$ delocalized electrons are partially attracted back
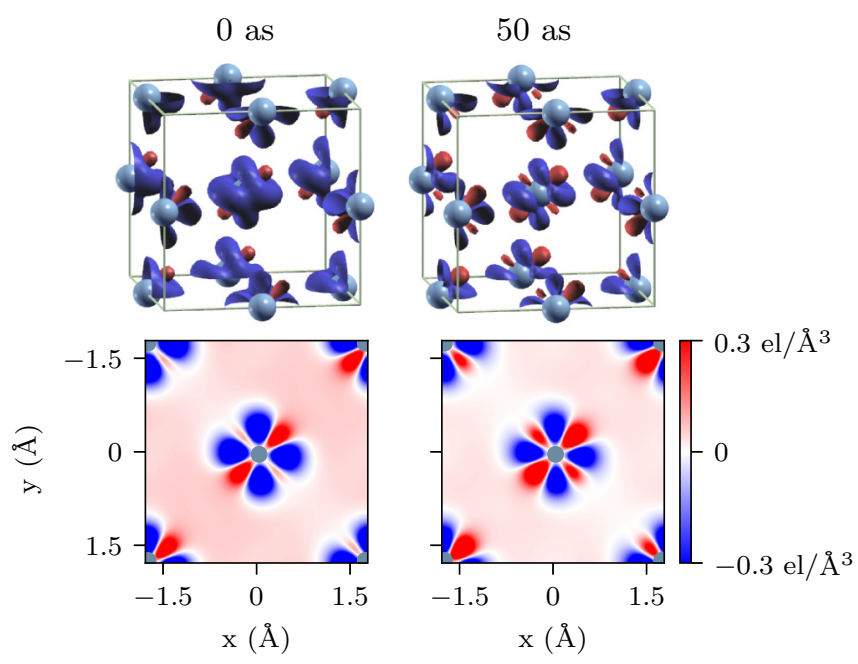

FIG. 2. Distribution of electron density change after an instant heating of electron subsystem in $\mathrm{Ni}$ at $0 \mathrm{~K}$ up to $50000 \mathrm{~K}$ shown at the initial stage and after 50 as. The 3D distributions as well as central cross sections are presented. Loss and gain of electrons are shown in blue and red, respectively. Atomic positions are indicated by gray circles or spheres. Screening imposes the reorganization of the electronic structure in 50 as.

to the nuclei and the $d$ states get slightly repopulated. All these relaxation processes happen on a timescale of 50 as defined by the upper limit of electron collision frequency in metals [47]. The ultrafast dynamics of the DOS response to electronic excitation can thus be neglected when laser pulses with duration longer than several femtoseconds are considered making valid the previously used approximations of the DOS instant change accordingly with an ad hoc temperature.

\section{B. $s p$ band vs $d$ band thermalization of $\mathrm{Ni}$}

The instant excitation of electrons to Fermi-Dirac distribution is a nonrealistic situation. An ultrashort laser pulse drives electrons out of thermal equilibrium. Later the photoexcited electrons thermalize to a Fermi-Dirac distribution. The ultrafast-laser-excited electron occupation numbers at different laser pulse intensities calculated using the TDDFT approach described in the previous section are shown in Fig. 3. The ground-state DOS and occupation number at zero temperature are also presented in the gray background.

Figure 3 shows transient occupation number of laserexcited electrons that deviates from a simple Fermi-Dirac distribution for given laser intensities. This demonstrates that electrons are not completely thermalized at such short durations. Particularly, the electron distribution is not symmetric with respect to the Fermi energy as a result of the nonsymmetric DOS. The DOS of Ni has a lot of $d$ electrons just below the Fermi energy that after absorbing photons form a hump above the Fermi energy. As the occupation number of the laser-excited metals can be approximated by a two-half of weighted Fermi-Dirac distributions, we observe that it can be remarkably well fitted by the following function:

$$
f=\frac{\beta}{1+\exp \left(\frac{\epsilon-\mu_{s p}}{k_{b} T_{s p}}\right)}+\frac{1-\beta}{1+\exp \left(\frac{\epsilon-\mu_{d}}{k_{b} T_{d}}\right)},
$$




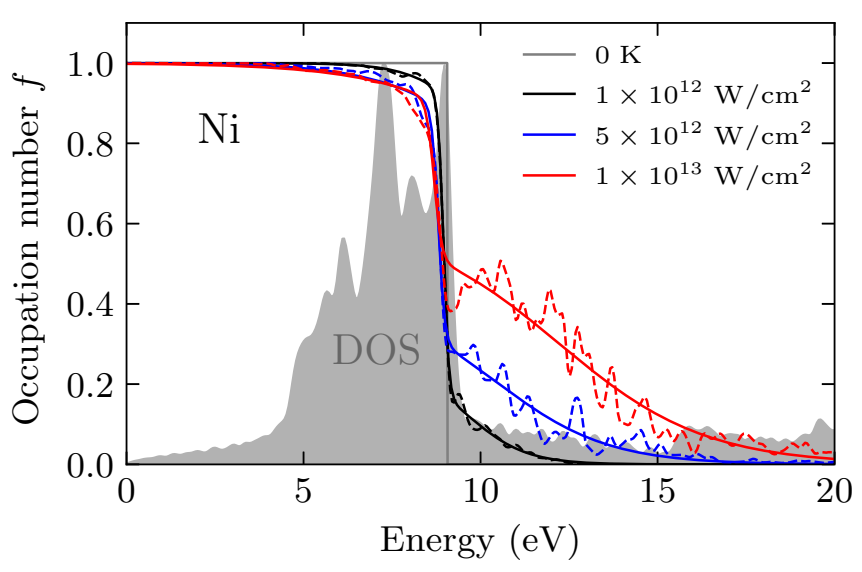

FIG. 3. Electron occupation number in Ni after the laser pulse with different peak intensities and $\tau_{F W H M}=7 \mathrm{fs}$ (dashed lines) and at $0 \mathrm{~K}$ (gray solid line). The fits to Eq. (9) are shown by solid lines with fitting parameters given in Table I. The normalized DOS of $\mathrm{Ni}$ at $0 \mathrm{~K}$ is shown by the gray-filled area.

showing the presence of two thermalized electron subsystems. The occupation number $f$ consists of two Fermi-Dirac distributions with their own temperature $T_{s p}, T_{d}$, and chemical potential $\mu_{s p}, \mu_{d}$. Within this dual system, the fraction of the electrons in the $s p$ subsystem is equal to $\beta$ and the fraction of the electrons in the $d$ subsystem is equal to $1-\beta$.

None of the fitting parameters remain constant as the laser intensity increases and their evolution is quite complex. For convenience, they are summarized in Table I. It is noteworthy that the related temperatures of both populations varies by one order of magnitude, $s p$ electrons being heated significantly higher that $d$ electrons. Moreover, influenced by the inherently changing photon absorption coefficient and the electron population fraction, the temperatures evolves nonlinearly with the peak intensity.

The spatial distribution of electron density change is mainly similar to the one observed in Fig. 2(a), i.e., there is an increase of density in the regions between atoms due to $d$ to $s p$ transitions and a redistribution of the density within the $d$ band near the ions. A certain correlation with the light polarization is observed but the effect is subtle, without modifying the main relaxation tendencies. For this reason it is not discussed here and a detailed report of the spatial electronic dynamics will be treated elsewhere.

TABLE I. Fitting parameters for electron occupation number in $\mathrm{Ni}$ in Fig. 3 according to Eq. (9). $T_{s p}$ and $\mu_{s p}$ are the temperature and chemical potential of $s p$ electrons, respectively. $T_{d}$ and $\mu_{d}$ are the temperature and chemical potential of $d$ electrons, respectively. $\beta$ is the fraction of $s p$ electrons and $1-\beta$ is the fraction of $d$ electrons. The relative fitting error for $\mu_{s p}, \mu_{d}$, and $\beta$ is around $1 \%$.

\begin{tabular}{lccrrr}
\hline \hline$I_{\text {las }}\left(\mathrm{W} / \mathrm{cm}^{2}\right)$ & $T_{s p}(\mathrm{~K})$ & $T_{d}(\mathrm{~K})$ & $\mu_{s p}(\mathrm{eV})$ & $\mu_{d}(\mathrm{eV})$ & $\beta$ \\
\hline $1 \times 10^{12}$ & $9600 \pm 100$ & $870 \pm 10$ & 9.63 & 8.95 & 0.25 \\
$5 \times 10^{12}$ & $18300 \pm 300$ & $1040 \pm 50$ & 10.44 & 8.81 & 0.41 \\
$1 \times 10^{13}$ & $23900 \pm 300$ & $1200 \pm 100$ & 12.21 & 8.69 & 0.60 \\
\hline \hline
\end{tabular}

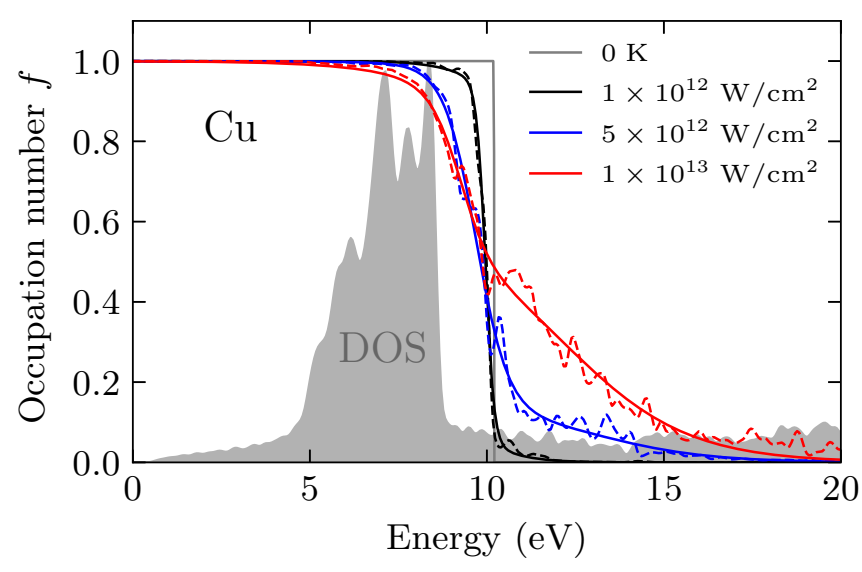

FIG. 4. Electron occupation number in $\mathrm{Cu}$ after the laser pulse with different peak intensities and $\tau_{F W H M}=7 \mathrm{fs}$ (dashed lines) and at $0 \mathrm{~K}$ (gray solid line). The fits to Eq. (9) are shown by solid lines with fitting parameters given in Table II. The normalized DOS of $\mathrm{Cu}$ at $0 \mathrm{~K}$ is shown by gray-filled area.

\section{C. $s p$ band vs $d$ band thermalization of partially filled $d$-band metals}

Compared to other transition metals, the particularity of $\mathrm{Ni}$ is that its $d$ band is located directly at the Fermi energy $E_{f}$ and can be easily depopulated by thermal excitations. In this subsection, we generalize the thermalization process to other transition metals. It was shown that the position of the $d$ band with respect to the Fermi energy and its filling play an important role in the modification of the DOS [12] and chemical potential [48] under electron excitation in transition metals. The overlapping between localized $s$ and $d$ bands also affect significantly this process. The $d$ band of the transition metals with BCC crystal structure is partially filled and is spread over several $\mathrm{eV}$ at both sides of the Fermi energy. Thus, we also analyze $\mathrm{Cu}$ and $\mathrm{Cr}$ that have characteristic $d$-band position, filling, and width and allow us to extend our conclusions obtained for $\mathrm{Ni}$.

Figure 4 shows the electron occupation number of $\mathrm{Cu}$ as a function of energy for different laser intensities. Effects similar to those for $\mathrm{Ni}$ are observed. The electrons from $d$-band located $\sim 2 \mathrm{eV}$ below the Fermi energy are excited above the Fermi energy forming a certain hump. This hump is however much less pronounced than the one in $\mathrm{Ni}$ at moderate laser intensities. This is due to the fact that the gap between the Fermi energy and the $d$-band edge in $\mathrm{Cu}$ is larger than photon energy $(1.55 \mathrm{eV})$ and thus higher laser intensity is needed to excite $d$ electrons. Additionally, there are more delocalized $s p$ electrons in $\mathrm{Ni}\left(3 d^{8} 4 s^{2}\right)$ than in $\mathrm{Cu}\left(3 d^{10} 4 s^{1}\right)$ that contribute stronger to the heating of the whole electron system. As a result, at lower laser intensity, mostly $s p$ electrons get excited as a result the occupation number behaves like if it has only one population of electrons. At higher intensity, however, the contribution of $d$ to $s p$ transitions becomes stronger leading to appearance of nonequilibrium features in the distribution function.

In case of Cr (Fig. 5), their is no gap between the Fermi energy and $d$ band that is spread along both sides of the Fermi level. As a result all kind of optical transitions take place 


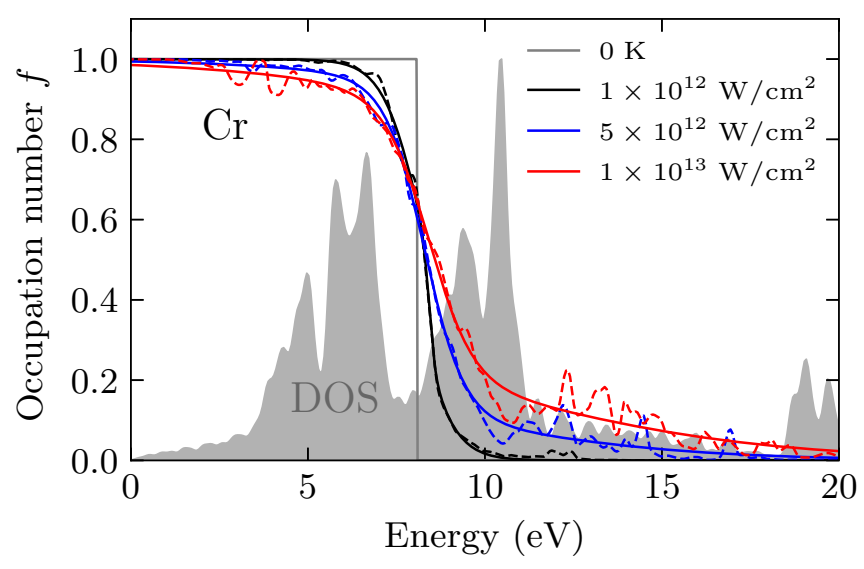

FIG. 5. Electron occupation number in $\mathrm{Cr}$ after the laser pulse with different peak intensities and $\tau_{F W H M}=7 \mathrm{fs}$ (dashed lines) and at $0 \mathrm{~K}$ (gray solid line). The fits to Eq. 9 are shown by solid lines with fitting parameters given in Table III. The normalized DOS of $\mathrm{Cr}$ at $0 \mathrm{~K}$ is shown by gray-filled area.

following the optical selection rules. Electrons photoexited from $d$ to $s p$ band and from $s p$ to $d$ band contribute equally to the electron population that can be more easily described by an equilibrium Fermi-Dirac distribution at low and high laser intensities.

To better understand the contribution of $s p$ and $d$ subpopulations to the photoexcited electron distribution, the obtained occupation numbers have been fitted to a double FermiDirac distribution using Eq. (9) for all three metals and laser intensities. The fitted values of temperatures, chemical potentials, and population fraction $\beta$ are given in Tables I-III. The relative fitting error is around $5 \%$ for all parameters. Note that chemical potentials of $\mathrm{Ni}, \mathrm{Cu}$, and $\mathrm{Cr}$ at zero temperature equal $9 \mathrm{eV}, 10.2 \mathrm{eV}$, and $8 \mathrm{eV}$, respectively. One can see that for all metals there are two populations that contribute to the distribution function: hot $s p$ electrons with a chemical potential $\mu_{s p}$ strongly shifted to the right from the Fermi energy at high intensities and high temperature $T_{s p}$ and cold $d$ electrons with almost constant chemical potential $\mu_{d}$ and lower temperature $T_{d}$. Additionally, with increasing laser intensity the fraction of each population changes. For $\mathrm{Ni}$, the fraction of $s p$ electrons $\beta$ gradually increases with intensity due to a higher rate of transitions of electrons from $d$ to $s p$ states. For $\mathrm{Cu}$, the fraction of $s p$ electrons stay almost constant at moderate laser intensities due to limited excitation of the $d$ band located much below the Fermi energy compared to

TABLE II. Fitting parameters for electron occupation number in $\mathrm{Cu}$ in Fig. 4 according to Eq. (9). $T_{d}$ and $\mu_{d}$ are the temperature and chemical potential of $d$ electrons, respectively. $\beta$ is the fraction of $s p$ electrons and $1-\beta$ is the fraction of $d$ electrons. The relative fitting error for $\mu_{s p}, \mu_{d}$, and $\beta$ is around $1 \%$.

\begin{tabular}{lccrrr}
\hline \hline$I_{\text {las }}\left(\mathrm{W} / \mathrm{cm}^{2}\right)$ & $T_{s p}(\mathrm{~K})$ & $T_{d}(\mathrm{~K})$ & $\mu_{s p}(\mathrm{eV})$ & $\mu_{d}(\mathrm{eV})$ & $\beta$ \\
\hline $1 \times 10^{12}$ & $8000 \pm 800$ & $1410 \pm 30$ & 9.8 & 10.0 & 0.10 \\
$5 \times 10^{12}$ & $18600 \pm 1400$ & $5200 \pm 100$ & 13.0 & 9.7 & 0.14 \\
$1 \times 10^{13}$ & $20100 \pm 300$ & $4900 \pm 300$ & 12.2 & 9.2 & 0.59 \\
\hline \hline
\end{tabular}

TABLE III. Fitting parameters for electron occupation number in $\mathrm{Cr}$ in Fig. 5 according to Eq. (9). $T_{d}$ and $\mu_{d}$ are the temperature and chemical potential of $d$ electrons, respectively. $\beta$ is the fraction of $s p$ electrons and $1-\beta$ is the fraction of $d$ electrons. The relative fitting error for $\mu_{s p}, \mu_{d}$ and $\beta$ is around $1 \%$.

\begin{tabular}{lccrrl}
\hline \hline$I_{\mathrm{las}}\left(\mathrm{W} / \mathrm{cm}^{2}\right)$ & $T_{s p}(\mathrm{~K})$ & $T_{d}(\mathrm{~K})$ & \multicolumn{1}{c}{$\mu_{s p}(\mathrm{eV})$} & $\mu_{d}(\mathrm{eV})$ & $\beta$ \\
\hline $1 \times 10^{12}$ & $5940 \pm 60$ & $1020 \pm 70$ & 8.1 & 8.4 & 0.71 \\
$5 \times 10^{12}$ & $35300 \pm 1500$ & $6500 \pm 100$ & 10.1 & 8.3 & 0.17 \\
$1 \times 10^{13}$ & $42500 \pm 1100$ & $7300 \pm 200$ & 10.9 & 8.4 & 0.3 \\
\hline \hline
\end{tabular}

$\mathrm{Ni}$ as discussed above. At higher laser intensity, when such excitation becomes possible the fraction of $s p$ electrons also increases as in the case of $\mathrm{Ni}$. In case of $\mathrm{Cr}$, the fraction of $s p$ electrons first decreases at higher laser intensity because for the given photon energy the transition of $s p$ electrons below the Fermi energy to the large $d$ band above the Fermi energy prevails. With further increase of intensity the excitation from $d$ to $s p$ band become possible and the fraction of $s p$ electrons increases again. Note also that temperatures of both populations are higher in $\mathrm{Cr}$ than in other metals for the same laser intensities because of the possibility of many different interand intraband transitions.

It is, thus, demonstrated that the $d$-block transition metals excited by an ultrashort laser pulse have two populations of electrons. The first one can be associated with excited $s p$-electrons that have much higher temperature $T_{s p}$ and temperature-dependent chemical potential that increases with laser intensity. The second one consists of $d$ electrons that stay relatively cold due to high spatial localization. The chemical potential of $d$ electrons does not vary with laser intensity and stays almost equal to the metal Fermi energy. For $\mathrm{Ni}$ and $\mathrm{Cu}$ at low temperature the fraction of $s p$ electrons $\beta$ is lower than the fraction of $d$ electrons $1-\beta$, that approximately correspond to the valence electronic configuration of the atoms, i.e., $3 d^{8} 4 s^{2}$ for $\mathrm{Ni}$ and $3 d^{10} 4 s^{1}$ for $\mathrm{Cu}$. With increasing laser intensity, $\beta$ increases because more electrons get excited from $d$ to $s p$ states. For $\mathrm{Cr}$ with partially-filled $d$-block and valence configuration of $3 d^{5} 4 s^{1}$ the situation is slightly different. Laser excitation leads to the strong redistribution of electrons inside the $d$ block and $s p$ to $d$ transitions at high laser intensities. Thus, the fraction of $s p$ electrons is higher at low laser intensities when electrons transit from $d$ to $s p$ states and it decreases at high laser intensities when $s p$ to $d$ transitions happen.

\section{Discussion on thermalization dynamics}

We have seen above that upon ultrafast laser excitation, TDDFT calculations reveal that electron populations of each orbital thermalized differently and the two subsystems share the total absorbed energy. The existence of two thermalized electron subsystems with different temperatures and chemical potentials has been previously assumed in the TTM study of laser-excited Ni [49]. A three-temperature model, describing the temperature evolution of the two subsystems and the lattice, has been developed to describe ultrafast laser-induced demagnetization of ferromagnetic materials [50]. Our study thus sheds light on the microscopic dynamical processes 
participating in the formation of the two subpopulations and show that this ultrafast phenomenon is universal to all transition metals with a fully or partially filled $d$ states. For longer pulse durations in hundreds of femtoseconds range, one could expect that the observed effect will vanish due to momentum and energy relaxation mediated by electron scattering through interband collision process and electron-phonon coupling.

We only followed the thermalization dynamics during the laser pulse because TDDFT in its current form cannot properly capture electron-electron relaxation processes when the external perturbation is turned off. As can be seen from Eqs. (2) and (3), when $\mathbf{A}=0$, ions do not move and occupation number is fixed to the initial one, and the Hamiltonian does not have time-dependent terms. The electron density and wave functions do not evolve in time making it impossible to describe electron thermalization. During the laser pulse when $\mathbf{A}(t) \neq 0$ the observed thermalization within each of the electron subsystems is dominated by electron-ion-photon collisions that have a characteristic time less than $1 \mathrm{fs}$ as was recently confirmed by a TDDFT simulation of optical properties of silicon [51].

The equilibration dynamics between the two subsystems after the laser pulse can only be studied if the TDDFT approach properly includes the electron-electron collisions. As it was shown in the first part of Sec. III, describing electron dynamics in $\mathrm{Ni}$ after an initial excitation, the possibility to include laser-excited electron relaxation in TDDFT through the time-dependent modification of fractional occupation numbers $f_{i}^{0}$ in the Hamiltonian should be explored. Additionally, the thermalization dynamics in the absence of the laser field can be obtained artificially by introducing strong ionic motion to the system and thus solving molecular dynamics equations together with Kohn-Sham equations [40].

\section{SUMMARY}

We applied TDDFT to analyze the electron relaxation and thermalization processes in different metals $(\mathrm{Ni}, \mathrm{Cu}, \mathrm{Cr})$ excited by an ultrashort laser pulse. TDDFT treats the electron dynamics in real time following the laser pulse from first principles at the atomic scale allowing for calculations without "two-temperature" or "constant density of states" approximations in the currently used models.
First, after instantly heating the electron system of $\mathrm{Ni}$ up to $50000 \mathrm{~K}$, we observed the evolution of the DOS as a result of "hot"-electron density spatial redistribution. The electron density changes in space are related to the modified ion screening and are observed within 50 as after the initial excitation. Interestingly, the TDDFT calculation converges to the results of the static DFT calculation demonstrating the effective electron-electron relaxation process.

We analyzed the behavior of electron occupation number in laser-excited $\mathrm{Ni}, \mathrm{Cu}$, and $\mathrm{Cr}$ as a function of laser intensity. After a 7-fs laser pulse two subsystems of electrons thermalized to two different temperatures can be observed. Localized electrons in $d$ block of these transition metals stay rather cold while the temperature of nearly free $s p$ electrons significantly increase due to laser excitation. Both temperatures increase with laser intensity. At high intensity, the chemical potential of $s p$ electrons also increases. Additionally, at higher laser intensity the fraction of the hot electrons is higher for $\mathrm{Ni}$ and $\mathrm{Cu}$, demonstrating the transition of electrons from $d$ to $s p$ states. In $\mathrm{Cr}$ the $d$ and $s p$ bands are nearly fully overlapped around the Fermi level and thus a more complex redistribution of the electrons between these states is observed. The relevance of TDDFT approach in given calculations is also discussed.

By their insights into the electronic dynamics, these original results redraw the limits of applicability of current approaches based on a single temperature for electrons within the classic two-temperature model. During the laser irradiation time, orbital-dependent temperatures are expected to impact transient optical response and subsequent energy distribution. Deepening the concept of an electronic structure dependence of the electron thermal distribution, accurate laser parameters can be defined for a specific irradiated transition metal. This will help to control the spatiotemporal energy confinement fostering the subsequent thermomechanical behavior. The followup phase transitions and ablative paths will determine surface structuring effects down to the nanoscale. This investigation provides original concepts to optimize laser-driven excitation dynamics and find routes for new energy relaxation channels.

\section{ACKNOWLEDGMENT}

This work was performed using HPC resources from GENCI-TGCC (Grant No. 2018-A0030907041).
[1] A. Y. Vorobyev and C. Guo, Laser Photonics Rev. 7, 385 (2013).

[2] K. Sugioka and Y. Cheng, Light: Sci. Appl. 3, e149 (2014).

[3] M. Malinauskas, A. Zukauskas, S. Hasegawa, Y. Hayasaki, V. Mizeikis, R. Buividas, and S. Juodkazis, Light Sci Appl. 5, e16133 (2016).

[4] B. Rethfeld, D. S. Ivanov, M. E. Garcia, and S. I. Anisimov, J. Phys. D: Appl. Phys. 50, 193001 (2017).

[5] A. Sommer, E. M. Bothschafter, S. A. Sato, C. Jakubeit, T. Latka, O. Razskazovskaya, H. Fattahi, M. Jobst, W. Schweinberger, V. Shirvanyan, V. S. Yakovlev, R. Kienberger, K. Yabana, N. Karpowicz, M. Schultze, and F. Krausz, Nature (London) 534, 86 (2016).
[6] G. Vampa, T. J. Hammond, N. Thiré, B. E. Schmidt, F. Légaré, C. R. McDonald, T. Brabec, and P. B. Corkum, Nature (London) 522, 462 (2015).

[7] G. L. Eesley, Phys. Rev. Lett. 51, 2140 (1983).

[8] C.-K. Sun, F. Vallée, L. H. Acioli, E. P. Ippen, and J. G. Fujimoto, Phys. Rev. B 50, 15337 (1994).

[9] R. H. M. Groeneveld, R. Sprik, and A. Lagendijk, Phys. Rev. B 51, 11433 (1995).

[10] S. I. Anisimov, B. L. Kapeliovich, and T. L. Perel'man, Zh. Eksp. Teor. Fiz. 66, 776 (1974) [Sov. Phys. JETP 39, 375 (1974)].

[11] J. Hohlfeld, S. S. Wellershoff, J. Güdde, U. Conrad, V. Jähnke, and E. Matthias, Chem. Phys. 251, 237 (2000). 
[12] E. Bevillon, J. P. Colombier, V. Recoules, and R. Stoian, Phys. Rev. B 89, 115117 (2014).

[13] E. Principi, E. Giangrisostomi, R. Cucini, F. Bencivenga, A. Battistoni, A. Gessini, R. Mincigrucci, M. Saito, S. Di Fonzo, F. D’Amico, A. Di Cicco, R. Gunnella, A. Filipponi, A. Giglia, S. Nannarone, and C. Masciovecchio, Struct. Dynam. 3, 023604 (2015).

[14] E. Bevillon, J. P. Colombier, V. Recoules, H. Zhang, C. Li, and R. Stoian, Phys. Rev. B 93, 165416 (2016).

[15] A. M. Brown, R. Sundararaman, P. Narang, A. M. Schwartzberg, W. A. Goddard, and H. A. Atwater, Phys. Rev. Lett. 118, 087401 (2017).

[16] E. Bévillon, R. Stoian, and J.-P. Colombier, J. Phys.: Condens. Matter 30, 385401 (2018).

[17] D. V. Minakov and P. R. Levashov, Phys. Rev. B 92, 224102 (2015).

[18] S. T. Murphy, Y. Giret, S. L. Daraszewicz, A. C. Lim, A. L. Shluger, K. Tanimura, and D. M. Duffy, Phys. Rev. B 93, 104105 (2016).

[19] Y. Giret, S. L. Daraszewicz, D. M. Duffy, A. L. Shluger, and K. Tanimura, Phys. Rev. B 90, 094103 (2014).

[20] S. T. Murphy, S. L. Daraszewicz, Y. Giret, M. Watkins, A. L. Shluger, K. Tanimura, and D. M. Duffy, Phys. Rev. B 92, 134110 (2015).

[21] E. Bevillon, J. P. Colombier, and R. Stoian, Appl. Surf. Sci. 374, 365 (2016).

[22] H. Zhang, C. Li, E. Bevillon, G. Cheng, J. P. Colombier, and R. Stoian, Phys. Rev. B 94, 224103 (2016).

[23] A. Giri, J. T. Gaskins, B. M. Foley, R. Cheaito, and P. E. Hopkins, J. Appl. Phys. 117, 044305 (2015).

[24] L. Waldecker, R. Bertoni, R. Ernstorfer, and J. Vorberger, Phys. Rev. X 6, 021003 (2016).

[25] J. Faure, J. Mauchain, E. Papalazarou, M. Marsi, D. Boschetto, I. Timrov, N. Vast, Y. Ohtsubo, B. Arnaud, and L. Perfetti, Phys. Rev. B 88, 075120 (2013).

[26] S. Rapp, M. Kaiser, M. Schmidt, and H. P. Huber, Opt. Express 24, 17572 (2016).

[27] J. Winter, S. Rapp, M. Schmidt, and H. P. Huber, Appl. Surf. Sci. 417, 2 (2017).

[28] S. A. Sato, K. Yabana, Y. Shinohara, T. Otobe, and G. F. Bertsch, Phys. Rev. B 89, 064304 (2014).

[29] C. Lian, S. B. Zhang, and S. Meng, Phys. Rev. B 94, 184310 (2016).

[30] B. Y. Mueller and B. Rethfeld, Phys. Rev. B 87, 035139 (2013).
[31] P. Maldonado, K. Carva, M. Flammer, and P. M. Oppeneer, Phys. Rev. B 96, 174439 (2017).

[32] T. Otobe, M. Yamagiwa, J.-I. Iwata, K. Yabana, T. Nakatsukasa, and G. F. Bertsch, Phys. Rev. B 77, 165104 (2008).

[33] G. Wachter, C. Lemell, and J. Burgdörfer, J. Phys.: Conf. Ser. 488, 012005 (2014).

[34] C. Race, The Modelling of Radiation Damage in Metals Using Ehrenfest Dynamics, Springer Theses (Springer, Berlin, Heidelberg, 2011).

[35] S. A. Sato, K. Yabana, Y. Shinohara, T. Otobe, K.-M. Lee, and G. F. Bertsch, Phys. Rev. B 92, 205413 (2015).

[36] N. A. Modine and R. M. Hatcher, J. Chem. Phys. 142, 20411 (2015).

[37] M. Lindenblatt, E. Pehlke, A. Duvenbeck, B. Rethfeld, and A. Wucher, Nucl. Instrum. Meth. B 246, 333 (2006).

[38] Z. Lin and R. E. Allen, J. Phys.: Condens. Matter 21, 485503 (2009).

[39] J. Bang, Y. Y. Sun, X.-Q. Liu, F. Gao, and S. B. Zhang, Phys. Rev. Lett. 117, 126402 (2016).

[40] W. Kang, S. Zhao, S. Zhang, P. Zhang, Q. F. Chen, and X.-T. He, Sci. Rep. 6, 20623 (2016).

[41] K. Yabana, T. Sugiyama, Y. Shinohara, T. Otobe, and G. F. Bertsch, Phys. Rev. B 85, 045134 (2012).

[42] D. Yu, L. Jiang, F. Wang, L. Qu, and Y. Lu, Appl. Phys. A 122, 494 (2016).

[43] X. Andrade, J. Alberdi-Rodriguez, D. A. Strubbe, M. J. T. Oliveira, Fernando Nogueira, A. Castro, J. Muguerza, A. Arruabarrena, S. G. Louie, A. Aspuru-Guzik, Angel Rubio, and M. A. L. Marques, J. Phys.: Condens. Matter 24, 233202 (2012).

[44] N. Troullier and J. L. Martins, Phys. Rev. B 43, 1993 (1991).

[45] J. P. Perdew, K. Burke, and M. Ernzerhof, Phys. Rev. Lett. 77, 3865 (1996).

[46] J. P. Perdew and A. Zunger, Phys. Rev. B 23, 5048 (1981).

[47] K. Eidmann, J. Meyer-ter-Vehn, T. Schlegel, and S. Hüller, Phys. Rev. E 62, 1202 (2000).

[48] Z. Lin, L. V. Zhigilei, and V. Celli, Phys. Rev. B 77, 075133 (2008).

[49] N. A. Inogamov, Y. V. Petrov, V. V. Zhakhovsky, V. A. Khokhlov, B. J. Demaske, S. I. Ashitkov, K. V. Khishchenko, K. P. Migdal, M. B. Agranat, S. I. Anisimov, V. E. Fortov, I. I. Oleynik, and C. Phipps, AIP Conf. Proc. 1464, 593 (2012).

[50] B. Y. Mueller and B. Rethfeld, Phys. Rev. B 90, 144420 (2014).

[51] S. A. Sato, Y. Shinohara, T. Otobe, and K. Yabana, Phys. Rev. B 90, 174303 (2014). 